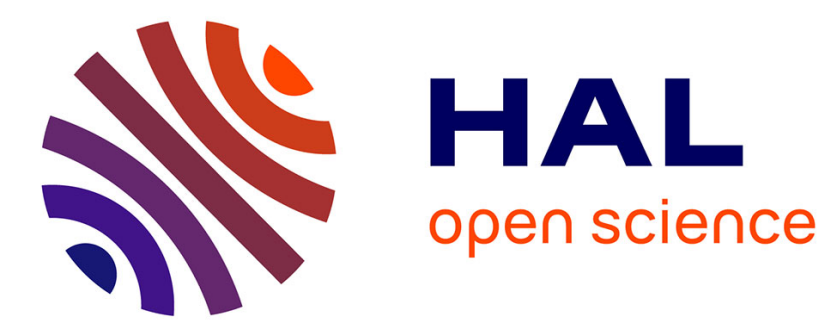

\title{
Différences inter-linguistiques dans l'apprentissage de la lecture
}

Johannes C Ziegler

\section{To cite this version:}

Johannes C Ziegler. Différences inter-linguistiques dans l'apprentissage de la lecture. Langue française, 2018, 3, pp.35-49. hal-01911663

\section{HAL Id: hal-01911663 \\ https://hal-amu.archives-ouvertes.fr/hal-01911663}

Submitted on 3 Nov 2018

HAL is a multi-disciplinary open access archive for the deposit and dissemination of scientific research documents, whether they are published or not. The documents may come from teaching and research institutions in France or abroad, or from public or private research centers.
L'archive ouverte pluridisciplinaire HAL, est destinée au dépôt et à la diffusion de documents scientifiques de niveau recherche, publiés ou non, émanant des établissements d'enseignement et de recherche français ou étrangers, des laboratoires publics ou privés. 


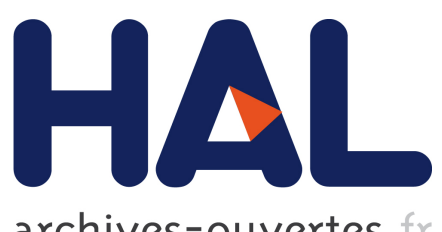

archives-ouvertes

\section{Différences inter-linguistiques dans l'apprentissage de la lecture}

Johannes C Ziegler

\section{To cite this version:}

Johannes C Ziegler. Différences inter-linguistiques dans l'apprentissage de la lecture. Langue française, Armand Colin, 2018, 3, pp.35-49. <hal-01911663>

\section{HAL Id: hal-01911663 https://hal-amu.archives-ouvertes.fr/hal-01911663}

Submitted on 3 Nov 2018

HAL is a multi-disciplinary open access archive for the deposit and dissemination of scientific research documents, whether they are published or not. The documents may come from teaching and research institutions in France or abroad, or from public or private research centers.
L'archive ouverte pluridisciplinaire HAL, est destinée au dépôt et à la diffusion de documents scientifiques de niveau recherche, publiés ou non, émanant des établissements d'enseignement et de recherche français ou étrangers, des laboratoires publics ou privés. 
Johannes C. Ziegler

Aix-Marseille Université \& CNRS, Laboratoire de Psychologie Cognitive (UMR 7290)

\section{Différences inter-linguistiques dans l'apprentissage de la lecture}

Beware of heard, a dreadful word That looks like beard and sounds like bird;

And dead: it's said like bed, not bead -

For goodness sake don't call it 'deed'. Watch out for meat and great and threat. They rhyme with suite and straight and debt.

(T.S. Watt, Manchester Guardian, 1954)

\section{LES MÉCANISMES FONDAMENTAUX DE L'APPRENTISSAGE DE LA LECTURE ${ }^{1}$}

La maitrise du langage oral précède normalement l'apprentissage lecture. Cela implique qu'avant d'apprendre à lire, l'enfant possède un « lexique mental ", « le trésor des mots » (Wortschatz), comme l'appelait W. Wundt (1832-1920), le fondateur de la psychologie expérimentale, dans lequel sont stockées la forme sonore (phonologique) des mots et leur signification (sémantique). L'apprentissage de la lecture ne fait que créer un nouveau chemin d'accès vers ce " trésor des mots » (Dehaene 2007). Pour cela, l'enfant dispose de deux mécanismes. Le premier, c'est l'apprentissage « par cœur» de la forme visuelle des mots. Ce mécanisme est limité (Grainger et al. 2012) car, dans la majorité des systèmes d'écriture, les

1. La préparation de cet article a bénéficié d'un financement de l'Agence Nationale de la Recherche (ANR13-APPR-0003] et du Programme Investissements d'Avenir Action « Innovation numérique pour l'excellence éducative », volet « Espaces de formation, de recherche et d'animation numérique » (e-FRAN). Cette recherche bénéficie également du soutien du Labex BLRI (ANR-11-LABX-0036), de l'Institut Convergence ILCB (ANR16-CONV-0002) et de l'Initiative d'Excellence d'Aix-Marseille Université (A*MIDEX). 
mots sont composés d'un petit nombre d'éléments - les lettres dans les systèmes alphabétiques - ce qui rend les mots visuellement peu distincts. Apprendre par cœur nécessiterait la mémorisation des milliers de combinaisons de ces mêmes éléments, combinaisons susceptibles d'être confondues. Ce type d'apprentissage serait comparable à la mémorisation d'un annuaire téléphonique : à moins de composer chacun des chiffres correctement et dans le bon ordre, la connexion échouera (Share 1995).

D'où l'importance du second mécanisme, le décodage ou déchiffrage, qui consiste à trouver pour chaque symbole le son correspondant. Le décodage est au cœur de l'apprentissage de la lecture dans toutes les langues alphabétiques (Ziegler \& Goswami 2005, 2006). Son efficacité repose sur deux bases : d'une part, les symboles de la majorité des systèmes d'écriture (p. ex. les lettres) correspondent de façon relativement systématique à des sons de la parole (les phonèmes) ; $d$ 'autre part, l'enfant connait la forme phonologique d'un grand nombre de mots avant l'apprentissage de la lecture. C'est ainsi que l'apprentissage d'un petit nombre de relations graphèmes-phonèmes (le b.a.-ba) permet à l'enfant de décoder des mots qu'il n'a jamais vus auparavant, mais dont il connait la forme phonologique et la signification. La mise en route de ce mécanisme nécessite d'abord un apprentissage systématique et explicite des principales règles ou associations grapho-phonologiques.

Dès lors que l'enfant a appris ces associations graphèmes-phonèmes élémentaires, il peut les appliquer pour décoder de nouveaux mots. C'est là que ce mécanisme prend tout son sens, car chaque fois qu'un décodage est réussi, c'està-dire qu'il active en mémoire la forme orale d'un mot déjà connu, le mécanisme du décodage est renforcé et une représentation orthographique du mot peut être créée et renforcée (Ziegler, Perry \& Zorzi 2014). L'apprentissage explicite (avec maitre) bascule alors vers l'apprentissage implicite (sans maitre). On parle alors d'un "mécanisme d'auto-apprentissage ", car c'est l'acte même de la lecture qui renforce la lecture (Share 1995 ; Ziegler, Perry \& Zorzi 2014). Ce mécanisme est puissant, puisqu'il permet à l'enfant de décoder des mots qu'il a déjà entendus, mais jamais lus auparavant, et cela de façon autonome. Le processus « d'autoapprentissage » permet l'automatisation de la lecture. Le mode sériel et laborieux du décodage est progressivement remplacé par le traitement de différentes unités psycholinguistiques (graphèmes, syllabes, morphèmes, mots) en parallèle, ce qui permet au lecteur expert de lire environ 3 à 4 mots par seconde avec une aisance et fluence qui donnent la fausse impression d'une lecture globale des mots (v. aussi Dehaene 2011 ; Rayner et al. 2001). 


\section{LES DIFFÉRENCES INTER-LANGUES}

La systématicité, dite " régularité » ou " consistance », des relations entre graphie et phonie varie d'un système d'écriture à l'autre (DeFrancis 1989). Certains systèmes d'écriture alphabétiques, comme l'italien, le grec ou le finnois, sont très réguliers dans le sens de la lecture (entre graphèmes et phonèmes) et de l'écriture (entre phonèmes et graphèmes). D'autres, comme le français ou l'allemand, sont réguliers dans le sens de la lecture, mais pas dans le sens de l'écriture (en français, par exemple, le même phonème peut souvent s'écrire de différentes façons : /o/ comme O, AU, EAU, OT, OP ; v. Ziegler et al. 1996). Enfin, certains systèmes d'écriture, comme notamment l'anglais, sont doublement irréguliers, à la fois dans le sens de la lecture (le graphème " $\mathrm{a}$ » se prononce différemment dans cat, was, saw, made et car), mais aussi dans le sens de l'écriture (le phonème /æ/ s'écrit de différentes façons dans can et men ; v. Ziegler et al. 1997).

Le niveau d'irrégularité peut être quantifié de différentes façons. Une manière approximative est de compter le nombre de graphèmes et de phonèmes. Si le nombre de phonèmes est supérieur au nombre de graphèmes, il est probable que le même graphème ait plusieurs prononciations. Si le nombre de phonèmes est inférieur au nombre de graphèmes, il est probable que le même phonème puisse s'écrire de différentes manières (mais cf. Tab. 1). Une deuxième façon est de faire un inventaire complet de toutes les règles graphèmes-phonèmes d'un système d'écriture et de vérifier combien de mots de la langue ne sont pas déchiffrables correctement en appliquant ces règles (Coltheart et al. 1993, 2001). Selon cette méthode, c'est donc le nombre de "violations » de ces règles qui détermine le niveau d'irrégularité, et non pas le nombre ou la complexité des règles elles-mêmes (Ziegler, Perry \& Coltheart 2000). Une troisième mesure, celle de la consistance, prend en compte simplement le nombre (ou la fréquence) d'associations possibles pour une même unité, comme par exemple, le nombre de prononciations pour une même lettre (a-/a/, a-/æ/, a-/e/, etc.) ${ }^{2}$. Il est alors crucial de définir sur quelle unité porte le calcul (la lettre individuelle, le graphème, la syllabe, l'attaque, la rime) et si le calcul est sensible à la position de l'unité dans le mot (p. ex. une même lettre peut avoir deux prononciations possibles, mais l'une apparait systématiquement au début du mot tandis que l'autre apparait systématiquement à la fin du mot). Le choix de l'unité est important pour les comparaisons inter-langues. Le Tableau 1 présente une estimation du degré de consistance de sept langues (finnois, hongrois, italien hollandais, portugais, français et anglais) en prenant en compte les relations lettres-sons pour la lettre initiale (Borgwaldt, Hellwig \& De Groot 2005).

2. Un indice fréquemment utilisé met en relation le nombre (ou la fréquence) d' « amis » (même prononciation) avec les nombre d' « amis » et d' « ennemis » [consistance = amis/(amis +ennemis)]. 
Lire-écrire : des savoirs scientifiques aux savoirs pratiques

Tableau 1 : Comparaison de 7 langues européennes en termes de nombre de lettres, nombre de phonèmes et degré de consistance (entropie a) pour la lettre initiale (plus l'entropie est faible, plus l'orthographe et régulier/consistant)

\begin{tabular}{|l|c|c|c|}
\hline Langue & Lettres & Phonèmes & Entropie \\
\hline Finnois & 29 & 30 & 0,00 \\
\hline Hongrois & 33 & 41 & 0,17 \\
\hline Italien & 30 & 30 & 0,20 \\
\hline Hollandais & 26 & 41 & 0,23 \\
\hline Portugais & 41 & 37 & 0,42 \\
\hline Français & 42 & 37 & 0,46 \\
\hline Anglais & 26 & 36 & 0,83 \\
\hline
\end{tabular}

a. L'entropie $(H)$ est une mesure de consistance. $H=\Sigma p_{i} \times \log _{2} 1 / p_{i}$, où $p_{1}$ est la probabilité de la première prononciation d'une lettre donnée, $p_{2}$ est la probabilité de la deuxième prononciation de la lettre (si une deuxième prononciation existe), et ainsi de suite pour toutes les prononciations possibles de cette lettre.

Une analyse plus fine du degré de consistance en fonction des unités linguistiques (consonne initiale, voyelle, consonne finale, attaque, rime) a été réalisée par R. Peereman et A. Content (1999) dans le sens de la lecture (graphie $\rightarrow$ phonie) et de l'écriture (phonie $\rightarrow$ graphie) pour le français et l'anglais (cf. Tab. 2).

Tableau 2 : Comparaison du degré de consistance en fonction de l'unité linguistique (consonne initiale, voyelle, consonne finale, attaque, rime) pour les mots monosyllabiques du français et de l'anglais

(d'après Peereman \& Content 1999)

\begin{tabular}{|l|c|c|c|c|}
\cline { 2 - 5 } \multicolumn{1}{c|}{} & \multicolumn{2}{c|}{ Graphie $\rightarrow$ Phonie } & \multicolumn{2}{c|}{ Phonie $\rightarrow$ Graphie } \\
\hline Unité & Anglais & Français & Anglais & Français \\
\hline Consonne initiale (C1) & 95 & 95 & 90 & 99 \\
\hline Voyelle (V) & 48 & 94 & 67 & 68 \\
\hline Consonne finale (C2) & 96 & 97 & 50 & 58 \\
\hline Attaque (C1V) & 57 & 96 & 74 & 73 \\
\hline Rime (VC2) & 91 & 98 & 67 & 58 \\
\hline
\end{tabular}

La consistance dans le sens de la lecture correspond à la proportion de mots contenant une unité orthographique donnée ayant la même prononciation que le mot cible par rapport au nombre total de mots contenant cette unité orthographique. La consistance dans le sens de l'écriture correspond à la proportion de mots contenant une unité phonologique donnée ayant la même orthographe que le mot cible par rapport au nombre total de mots contenant cette unité phonologique. Comme le montre le Tableau 2, le français est bien plus régulier que l'anglais dans le sens de la lecture, notamment pour la voyelle et l'attaque 
(Ziegler et al. 1996). En revanche, dans le sens de l'écriture, le niveau d'irrégularité est comparable, notamment pour la voyelle. Il est intéressant de noter que la consistance de l'anglais, mais pas celle du français, augmente pour les unités plus grandes, notamment la rime en lecture et l'attaque en écriture par rapport à la voyelle seule (cf. les implications pour un modèle général de l'apprentissage de la lecture infra).

\section{LES DIFFÉRENCES INTER-LANGUES DANS L'APPRENTISSAGE DE LA LECTURE}

Le décodage jouant un rôle central dans l'apprentissage de la lecture (Ziegler \& Goswami 2005), il en résulte la prédiction forte que le degré de consistance $d^{\prime}$ 'un système d'écriture devrait déterminer très fortement la facilité et la vitesse avec laquelle la lecture est apprise et maitrisée (Ziegler \& Goswami 2006). Plusieurs recherches inter-langues ont testé cette prédiction (Bruck, Genesee \& Caravolas 1997; Goswami, Gombert \& Frace de Barrera 1998; Wimmer \& Goswami 1994). Par exemple, U. Goswami, J. E. Gombert et L. Fraca de Barrera (1998) ont étudié la lecture de pseudomots (capacité de décodage) dans trois langues (français, anglais et espagnol) chez des enfants âgés de 7 à 9 ans. Les résultats ont montré que les enfants anglais de 7 ans avaient un niveau de lecture, estimé par le pourcentage de pseudomots lus correctement, bien inférieur (21\%) à celui des enfants espagnols du même âge (90\%). Le niveau de lecture des enfants français était intermédiaire (63\%). L'étude de M. Bruck et ses collègues (1997) a confirmé ces résultats avec des enfants canadiens francophones et anglophones âgés de 6 à 7 ans. À la fin de la première année scolaire, les enfants anglophones ne lisaient que $51 \%$ des mots et $38 \%$ des pseudomots correctement tandis que les francophones lisaient $76 \%$ des mots et $62 \%$ des pseudomots.

Une comparaison particulièrement révélatrice est celle entre l'allemand et l'anglais, deux langues germaniques, ayant un système orthographique et phonologique assez similaire. Toutefois, ces langues se distinguent par la régularité des relations entre graphèmes et phonèmes (v. Ziegler, Perry \& Coltheart 2000). Par exemple, les mots ball (ballon), park (parc) et land (pays) s'orthographient de la même façon dans les deux langues, cependant le graphème " $a$ " qui se prononce toujours de la même manière en allemand possède une prononciation différente dans chacun de ces mots en anglais. En utilisant des mots quasi identiques dans les deux langues (des cognates), contrôlant ainsi au maximum les facteurs visuels, sémantiques et articulatoires, U. Frith, H. Wimmer et K. Landerl (1998) ont étudié le niveau et l'évolution des performances de lecture pour les enfants de 7 à 12 ans en Angleterre et en Allemagne. Avec ces stimulus fortement appariés, ils ont d'abord répliqué le fait que les enfants anglais avaient un niveau de lecture et de décodage phonologique (lecture de pseudomots) bien inférieur à celui des enfants allemands, notamment au début de l'apprentissage. Ensuite, 
ils ont montré qu'il fallait quelques années supplémentaires d'enseignement aux élèves anglais pour atteindre le niveau des enfants allemands (cf. Fig. 1).

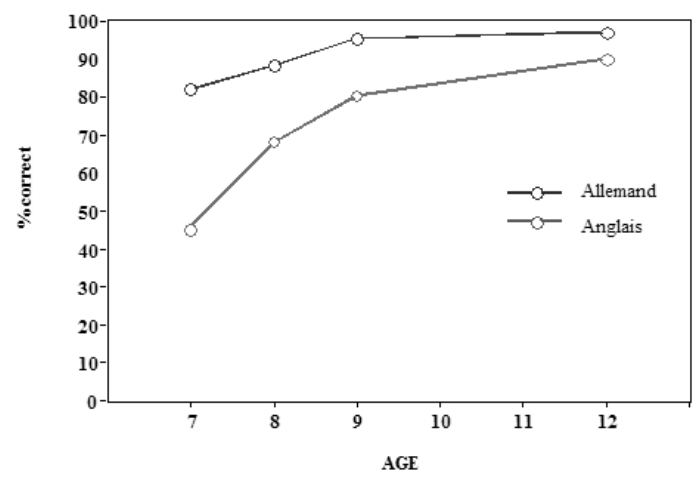

Figure 1 : Niveau de lecture des pseudomots pour les enfants allemands et anglais de 7 à 12 ans (d'après Frith, Wimmer \& Landerl 1998)

Des résultats similaires ont été obtenus chez des jeunes lecteurs allemands et anglais de 7 à 9 ans, appariés sur l'âge de lecture, qui devaient lire à voix haute des pseudomots sans voisins orthographiques et phonologiques (Goswami et al. 2001). Ces stimuli nécessitaient donc un décodage graphème-phonème. Les résultats ont montré que les jeunes enfants anglais de 7 ans avaient beaucoup plus de mal à lire ces stimuli que les Allemands de même âge (performances de décodage de l'ordre de $20 \%$ contre près de $80 \%$ ).

Pour généraliser ces résultats à l'échelle de l'Europe, le niveau de lecture et les capacités de décodage phonologique (lecture des pseudomots) ont été mesurés à la fin de la première année scolaire dans quatorze pays différents (les données obtenues sont décrites en détail dans un article de Seymour, Aro \& Erskine 2003). Les résultats ont montré que le niveau de lecture varie d'une langue à l'autre après seulement une année d'enseignement, avec de meilleures performances pour les langues qui ont une orthographe relativement consistante (le grec, le finlandais, l'allemand) que pour celles qui ont une orthographe moins consistante (particulièrement l'anglais). Pour illustrer ces résultats, la Figure 2 met en correspondance le niveau de précision en lecture (\% d'erreurs) et la vitesse à la fin de la première année scolaire avec l'indice de consistance (ici « entropie ») pour le finnois, hongrois, italien hollandais, portugais, français et anglais. Comme le montre cette Figure 2, le taux d'erreurs et la vitesse de lecture à la fin de la première année sont quasi parfaitement prédits par le degré de consistance de la langue. Cette concordance entre le degré de consistance et le niveau de lecture est en faveur de l'idée que la consistance des relations grapho-phonémiques détermine fortement la facilité avec laquelle le mécanisme de décodage peut se mettre en place et explique pourquoi un enfant finlandais 
apprend les bases de la lecture en quelques semaines, tandis qu'un enfant anglais a besoin de beaucoup plus de temps pour arriver au même résultat.

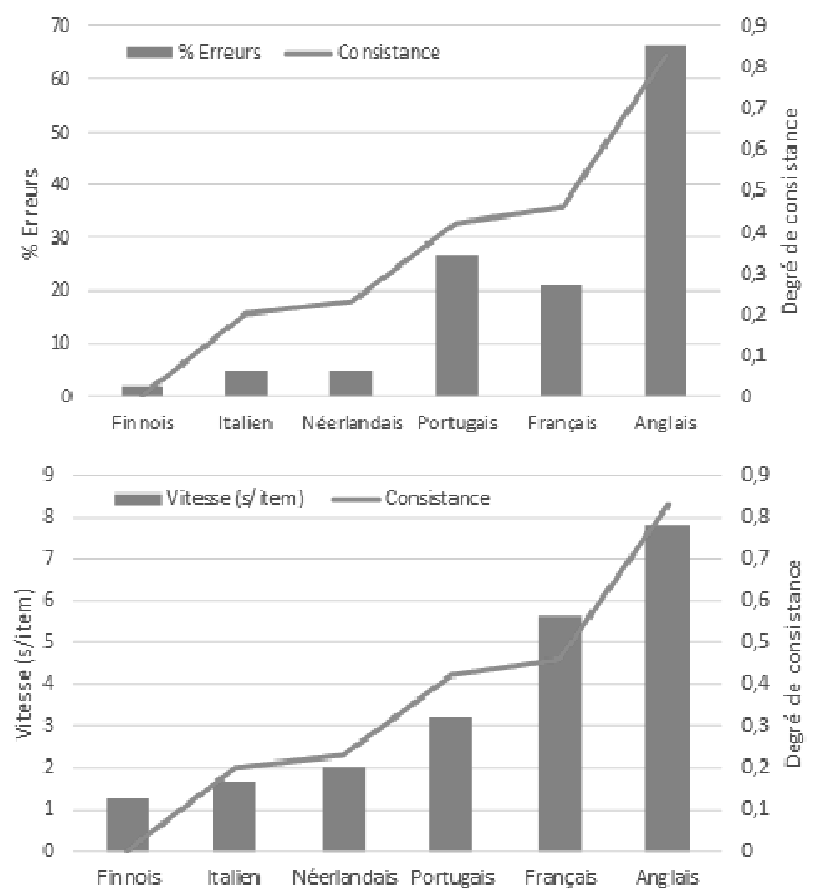

Figure 2 : Relation entre le niveau de lecture (\% d'erreurs) et la vitesse de lecture à la fin de la première année scolaire avec le degré de consistance

\section{PRÉDICTEURS UNIVERSELS DE L'APPRENTISSAGE DE LA LECTURE}

Une question importante concerne les variables qui sont prédictives de l'apprentissage de la lecture et de ses dysfonctionnements. L'idée générale est de savoir si les « prédicteurs » de l'apprentissage de la lecture, notamment la conscience phonémique (souvent évaluée par des épreuves de suppressions de phonèmes), l'intelligence non verbale, le vocabulaire, la dénomination rapide et la mémoire phonologique, ont le même poids dans toutes les langues ou si la nature du système d'écriture ( $p$. ex. la consistance des relations graphème-phonème) module leur influence respective. Pour répondre à cette question, nous avons réalisé une étude sur 1200 enfants issus de six pays différents (Ziegler et al. 2010).

Deux résultats importants ressortent d'une analyse dans laquelle toutes les variables ont été utilisées de façon simultanée dans une régression linéaire 
pour prédire la lecture en termes de fluence (mots correctement lus en une minute). Premièrement, comme le montre le Tableau 3, la capacité d'isoler les phonèmes est le meilleur prédicteur du niveau de lecture, aussi bien dans les langues qui ont une orthographe transparente (comme le finlandais) que dans celles qui ont une orthographe moins transparente (comme le français). La dénomination rapide ${ }^{3}$ a une influence moins systématique sans doute à cause de sa forte corrélation avec la conscience phonémique qui «absorbe » une partie importante de la variance. Le niveau en vocabulaire est important au moins pour le français et le finnois. Enfin, ni la mémoire phonologique ni l'intelligence non verbale ne contribuent significativement en présence des autres variables (pour une réplication, cf. infra Landerl et al. 2013). Le second résultat important est que le poids de certains facteurs est modulé par la transparence de la langue. La capacité de manipuler les phonèmes a une influence moins importante quand l'orthographe est transparente sans doute parce que la mise en route du décodage est facilitée par cette transparence. Dans le même cas de figure, le vocabulaire a une influence plus importante probablement parce que la facilité du décodage dans une orthographe transparente permet à d'autres facteurs, comme le langage oral, de mieux s'exprimer, et donc d'expliquer des parts de variance plus importantes.

Tableau 3 : Coefficients de corrélation d'une régression simultanée prédisant le niveau de lecture (fluence) de $\mathbf{1} 200$ élèves à la fin du grade 2 à partir de cinq variables langagières et cognitives (selon Ziegler et al. 2010)

\begin{tabular}{|l|c|c|c|c|c|}
\hline \multicolumn{1}{|c|}{ Variable } & $\begin{array}{c}\text { Finlande } \\
(\mathbf{n}=166)\end{array}$ & $\begin{array}{c}\text { Hongrie } \\
(\mathbf{n}=139)\end{array}$ & $\begin{array}{c}\text { Pays-Bas } \\
(\mathbf{n}=597)\end{array}$ & $\begin{array}{c}\text { Portugal } \\
(\mathbf{n}=182)\end{array}$ & $\begin{array}{c}\text { France } \\
(\mathbf{n}=181)\end{array}$ \\
\hline $\begin{array}{l}\text { Conscience } \\
\text { Phonémique }\end{array}$ & $0,31 * * *$ & $0,49 * * *$ & $0,41 * * *$ & $0,54 * * *$ & $0,53^{* * *}$ \\
\hline $\begin{array}{l}\text { Dénomination } \\
\text { Rapide }\end{array}$ & 0,08 & $0,30 * * *$ & $0,12^{* *}$ & $0,15^{*}$ & 0,11 \\
\hline $\begin{array}{l}\text { Mémoire } \\
\text { Phonologique }\end{array}$ & 0,03 & 0,04 & 0,04 & $-0,01$ & 0,05 \\
\hline Vocabulaire & $0,32 * * *$ & $-0,12$ & 0,06 & $-0,01$ & $0,14^{*}$ \\
\hline $\begin{array}{l}\text { Intelligence } \\
\text { Non verbale }\end{array}$ & $-0,05$ & $-0,10$ & 0,03 & 0,09 & 0,03 \\
\hline $\begin{array}{l}\text { Variance totale } \\
\text { expliquée (R2) }\end{array}$ & .25 & .34 & .23 & .41 & .38 \\
\hline
\end{tabular}

Dans une étude similaire, K. Landerl et al. (2013) ont comparé des compétences cognitives et langagières d'environ 1000 enfants dyslexiques et 1000 enfants témoins âgés de 8 à 12 ans, originaires de huit pays européens.

3. Le sujet est présenté avec une feuille avec une dizaine de lignes comportant 5 images d'objets (ou couleurs, chiffres, lettres) répétées de façon aléatoire et il doit dire son nom aussi rapidement que possible. On mesure le taux d'erreurs et surtout le temps mis à dénommer l'ensemble des objets sur la feuille. 
Comme J. C. Ziegler et al. (2010), ils ont testé dans quelle mesure, d'une part, divers facteurs cognitifs et langagiers permettaient de prédire le niveau de lecture et, d'autre part, si ces facteurs variaient d'un pays à l'autre en fonction du système orthographique. Les variables étaient similaires à celles de J. C. Ziegler et al. (2010), notamment la suppression de phonèmes, la dénomination rapide et la mémoire phonologique. Les résultats ont montré que la conscience phonémique et la dénomination rapide étaient des prédicteurs forts de la dyslexie et que la mémoire phonologique n'avait qu'un poids mineur. L'influence de la conscience phonémique et de la dénomination rapide s'est révélée plus importante dans des langues ayant une orthographe peu consistante. Il est intéressant de noter que le modèle statistique livrait de meilleures prédictions dans les langues ayant une orthographe peu consistante probablement à cause d'une variabilité plus importante. De manière générale, dans les langues ayant une orthographe consistante, le niveau de lecture est généralement plus élevé (Seymour, Aro \& Erskine 2003), même pour les enfants dyslexiques (Ziegler et al. 2003).

\section{UN MODĖLE GÉNÉRAL DE L'APPRENTISSAGE DE LA LECTURE}

Avec U. Goswami de l'Université de Cambridge, nous avons développé une théorie de l'apprentissage de la lecture capable d'expliquer les différences interlangues (Ziegler \& Goswami 2005).

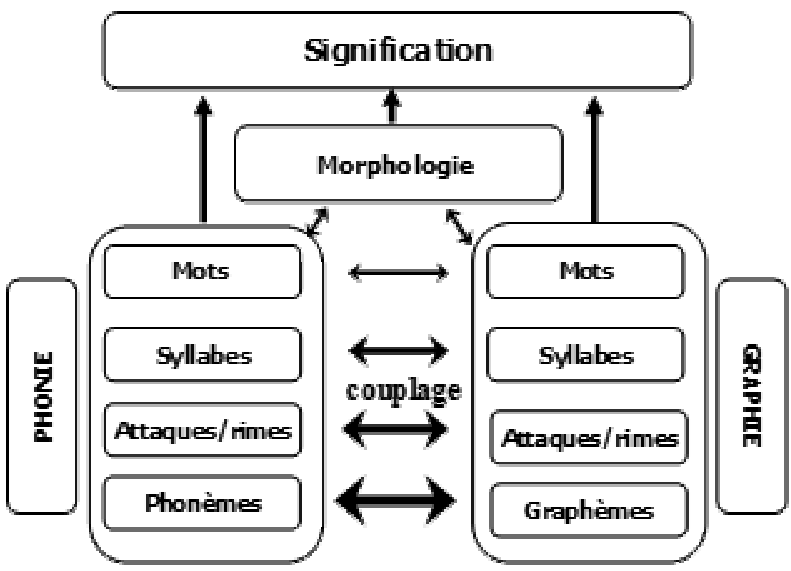

Figure 3 : La «psycholinguistic grain size theory » de Ziegler \& Goswami $(2005,2006)$

Au cœur de l'apprentissage de la lecture, dans toutes les langues, se trouve le couplage entre graphie et phonie. Ce couplage se fait à différents niveaux psycholinguistiques : graphèmes-phonèmes, rimes, syllabes, mots entiers. Dans 
une langue dont les unités de petite taille (graphèmes et phonèmes) sont relativement régulières (l'allemand par exemple), le couplage peut être initié à ce niveau. L'apprentissage d'un petit nombre de correspondances graphèmes-phonèmes suffit alors pour accéder au lexique phonologique. En revanche, dans une langue dont les unités de petite taille sont irrégulières (l'anglais par exemple), les enfants ne peuvent pas décoder les mots sur la seule base de ces petites correspondances. Ils sont donc obligés d'utiliser des unités de plus grande taille (p. ex. rime, syllabe, mot entier). Cependant, plus la taille des unités est grande, plus il y a d'unités à mémoriser.

Toutefois, alors qu'en finlandais ou en italien, dans la mesure où il n'y a que peu de correspondances à apprendre ( 30 lettres $\rightarrow 30$ phonèmes), l'apprentissage du décodage est typiquement réalisé en quelques mois, le problème est plus complexe pour le français, en particulier pour les voyelles. Cela est dû au fait qu'il n'y a que 6 lettres dans l'alphabet permettant d'écrire les 12 à 16 voyelles du français (Peereman \& Sprenger-Charolles 2018 ce volume) : il a donc été nécessaire d'utiliser soit une lettre avec un signe diacritique pour différencier <é $>$ de $<\mathrm{e}>$, soit plusieurs lettres pour différencier /u/ $(<\mathrm{ou}>)$ de /y/ $(<\mathrm{u}>)$, tout comme les voyelles nasales $(<\mathrm{an}\rangle,<\mathrm{on}\rangle,<\mathrm{un}\rangle,<\mathrm{in}\rangle)$ des voyelles orales $(<\mathrm{a}\rangle,<\mathrm{o}\rangle,\langle\mathrm{u}\rangle$, $<\mathrm{i}>$ ). Par conséquent, il faut plus de temps pour apprendre à lire en français à cause, entre autres, du nombre important des correspondances à apprendre. Il faut également un enseignement plus systématique et mieux construit (Ruiz et al. 2017 ; Sprenger-Charolles 2017).

Les prédictions de cette théorie ont été validées dans de nombreuses études. Par exemple, contrairement aux enfants allemands, les enfants anglais de 7 à 9 ans montrent un " effet de pseudohomophone » facilitateur qui reflète le fait qu'un pseudomot, comme roze, qui partage la forme phonologique du vrai mot rose, est lu plus rapidement à voix haute qu'un contrôle orthographique, comme rofe (Goswami et al. 2001). Ce résultat montre que les enfants anglais cherchent davantage à intégrer les informations lexicales (" unités de grande taille ») lors de la lecture à voix haute.

Dans une autre étude, U. Goswami et ses collaborateurs (2003) ont montré que les Anglais adaptent le type de décodage (par unités de petite ou de grande taille) en fonction du mode de présentation : les mêmes stimuli sont mieux lus lorsque leur présentation est bloquée par « taille d'unités » que lorsque la présentation est mélangée. En revanche, les enfants allemands passent invariablement par un décodage par unités de petite taille et ne montrent donc aucun bénéfice du mode de présentation.

De plus, à l'aide de l'enregistrement des mouvements oculaires, A. K. Rau et al. (2015) ont montré que les enfants allemands effectuent un traitement préférentiel par « unités de petite taille » dès les premières fixations. Cet effet est présent chez les enfants anglais uniquement pour la « relecture » (au deuxième passage) probablement lorsqu'ils rencontrent des difficultés. 
En outre, dans une étude portant sur le lecteur expert, J. C. Ziegler et ses collaborateurs (2001) ont montré que les Allemands continuent à favoriser un traitement par unités de petite taille comme l'indique l'effet de la longueur des mots particulièrement marqué chez eux, mais pas chez les Anglais. En revanche, les Anglais continuent à montrer une forte sensibilité pour les unités de grande taille comme l'indique l'effet de voisinage de rime.

Enfin, il a été montré chez les enfants bilingues que la transparence/granularité de l'orthographe de la première langue (L1) influence la manière dans laquelle les enfants traitent la seconde langue (L2). Ainsi, dans une étude récente, M. Lallier, J. Acha et M. Carreiras (2016) ont comparé des enfants bilingues français/basques et espagnols/basques dans des tâches de lecture (dans leur langue commune, le basque), de conscience phonémique et d'empan visuel (Bosse, Tainturier \& Valdois 2007). Comme prédit par la " psycholinguistic grain size theory », les enfants bilingues espagnols/basques ont montré de meilleures performances en lecture de pseudomots (décodage) et de conscience phonémique que les bilingues français/basques. En revanche, les enfants bilingues français/basques ont montré un empan visuel plus étendu, donc un traitement visuo-attentionnel plus sensible aux unités de grande taille que celui des enfants espagnols/basques.

\section{CONCLUSIONS}

Depuis les années 70, le domaine de l'apprentissage de la lecture dans une écriture alphabétique a été dominé par des études et théories anglophones (Bradley \& Bryant 1983 ; Coltheart et al. 1993, 2001 ; Harm \& Seidenberg 1999 ; Plaut et al. 1996 ; Rayner et al. 2001 ; Seidenberg \& McClelland 1989). L'ironie du sort est que la langue anglaise $a$ un système orthographique très inconsistant et elle est aujourd'hui décrite comme " l'exception plutôt que la règle » :

a outlier orthography... whose extreme ambiguity of English spelling-sound correspondence has confined reading science to an insular, Anglocentric research agenda addressing theoretical and applied issues with limited relevance for a universal science of reading. (Share, $2008: 584$ )

Sans aller aussi loin que D. L. Share (2008), il convient de réévaluer certaines idées reçues à la lumière des résultats issus des comparaisons inter-langues.

Premièrement, la manière d'enseigner la lecture ne provoque des débats animés que pour des langues qui ont une orthographe peu consistante, notamment pour l'anglais et, mais dans une moindre mesure, le français. La forte irrégularité de l'orthographe de l'anglais a conduit certains chercheurs et didacticiens anglosaxons à condamner la méthode du décodage au profit d'une méthode globale qui interdisait toute forme de déchiffrage (Goodman 1967). Les conséquences de l'enseignement global se sont avérées catastrophiques avec une chute importante du niveau de lecture à la fin des années 80 . Le gouvernement américain 
demande alors un rapport au National Reading Panel (2000) qui fait état d'une centaine d'études montrant que l'enseignement systématique du décodage est plus efficace que son enseignement non systématique, notamment pour les enfants à risque de difficultés d'apprentissage de la lecture. En 1997, l'Angleterre à son tour met en place un programme d'éducatif radical et ambitieux (National Literacy Strategy) qui après dix ans montre des effets spectaculaires (Department for Education 2011) et qui sont aujourd'hui confirmés par les récentes évaluations PIRLS (DEPP 2017). Dans ce programme, l'on trouve notamment un curriculum détaillé pour l'apprentissage de la lecture qui met l'accent sur un enseignement systématique du décodage et présente un guide pour y parvenir (Department for Education 2013). Si le débat des méthodes (analytique vs globale) avait un certain sens dans le milieu anglo-saxon, ce débat n'a pas lieu d'être dans des langues qui ont une orthographe régulière, car les enfants peuvent pleinement profiter des relations systématiques entre graphèmes et phonèmes.

Deuxièmement, la conception assez « anglocentrique » selon laquelle il existe une « voie phonologique » lente, laborieuse et accessoire qui doit être remplacée par un tout autre processus, une voie « orthographique » directe, rapide et efficace (Coltheart et al. 1993) reflète clairement un biais lié à l'irrégularité du système orthographique (Share 2008). Il est aujourd'hui clair que le décodage phonologique est au cœur de l'apprentissage de la lecture dans toutes les langues, car c'est lui qui permet la mise en place du mécanisme de l'auto-apprentissage même en anglais (Ziegler, Perry \& Zorzi 2014). Bien qu'il soit initialement lent et sériel, le mécanisme du décodage se renforce rapidement pour finir par opérer de façon parallèle sur différentes tailles d'unités. L'idée selon laquelle il laisserait la place à un mécanisme purement orthographique, et non phonologique, n'est pas étayée ni par des modèles inter-langues de la lecture (Frost 1998 ; Share 2008 ; Ziegler \& Goswami 2005), ni par des données empiriques issues de la psycholinguistique (Braun et al. 2009 ; Sprenger-Charolles et al. 2003 ; Van Orden 1987 ; Ziegler, Van Orden \& Jacobs 1997) ou de l'imagerie cérébrale (Rueckl et al. 2015).

De la même façon, l'idée selon laquelle il existerait deux sous-types d'enfants dyslexiques ayant la même importance et prévalence dans la population (Castles \& Coltheart 1993) - l'un étant le résultat d'un déficit de la " voie orthographique " (dyslexie de surface) et l'autre d'un déficit de la « voie phonologique »-s'est avérée partiellement fausse (Manis et al. 1996 ; Sprenger-Charolles et al. 2011). En effet, la majorité des enfants dyslexiques souffrent de troubles phonologiques (Saksida et al. 2016 ; White et al. 2006 ; Ziegler et al. 2008) et les cas de dyslexie de surface semblent moins fréquents, et plutôt liés à un retard dans le développement, ainsi qu'à des phénomènes de compensation, plutôt qu'à un déficit fondamental. 


\section{Références bibliographiques}

Borgwaldt S. R., Hellwig F. M. \& De Groot A.M.B. (2005), "Onset entropy matters - letter-tophoneme mappings in seven languages", Reading and Writing 18 (3), 211-229.

Bosse M.-L., TAINTURIER M. J. \& VAldoIS S. (2007), " Developmental dyslexia: The visual attention span deficit hypothesis", Cognition 104 (2), 198-230.

BRADLEY L. \& BRYANT P. E. (1983), "Categorizing sounds and learning to read: A causal connection”, Nature 301, 419-421.

Braun M. et al. (2009), "Pseudohomophone effects provide evidence of early lexico-phonological processing in visual word recognition", Human Brain Mapping 30 (7), 1977-1989.

BRuck M., Genesee F. \& Caravolas M. (1997), “A cross-linguistic study of early literacy acquisition", in B. A. Blachman (ed.), Foundations of Reading Acquisition and Dyslexia: Implications for Early Intervention, Mahwah (NJ), Erlbaum, 145-162.

Castles A. \& Coltheart M. (1993), “Varieties of developmental dyslexia”, Cognition 47 (2), $149-180$

COLTHEART M. et al. (1993), "Models of reading aloud: Dual-route and parallel-distributedprocessing approaches", Psychological Review 100 (4), 589-608.

COLTHEART M. et al. (2001), "DRC: A dual route cascaded model of visual word recognition and reading aloud", Psychological Review 108 (1), 204-256.

DeFrancis J. (1989), Visible Speech: The Diverse Oneness of Writing Systems, Honolulu, University of Hawaii Press.

Dehaene S. (2007), Les neurones de la lecture, Paris, Odile Jacob.

DEHAENE S. (2011), Apprendre à lire : des sciences cognitives à la salle de classe, Paris, Odile Jacob.

DePARTMENT FOR EDUCATION (2011), The National Strategies 1997-2011: A brief summary of the impact and effectiveness of the National Strategies. [en ligne]

DEPARTMENT FOR EDUCATION (2013), National curriculum in England: Framework for key stages 1 to 4. [en ligne]

DEPP (2017), "PIRLS 2016 - Évaluation internationale des élèves de CM1 en compréhension de l'écrit - Évolution des performances sur quinze ans ", Note d'information 17.24, Décembre 2017.

FRITH U., WiMmeR H. \& LANDERL K. (1998), "Differences in phonological recoding in German- and English-speaking children", Scientific Studies of Reading 2 (1), 31-54.

FROST R. (1998), “Toward a strong phonological theory of visual word recognition: True issues and false trails", Psychological Bulletin 123 (1), 71-99.

Goodman K. S. (1967), “Reading: A psycholinguistic guessing game”, Journal of the Reading Specialist - Literacy Research and Instruction 6 (4), 126-135.

Goswami U., Gombert J. E. \& Fraca DE BARReRA L. (1998), “Children's orthographic representations and linguistic transparency: Nonsense word reading in English, French, and Spanish", Applied Psycholinguistics 19 (1), 19-52.

GoswAMI U. et al. (2001), "Pseudohomophone effects and phonological recoding procedures in reading development in English and German", Journal of Memory and Language 45 (4), 648-664.

GoswamI U. et al. (2003), "Nonword reading across orthographies: How flexible is the choice of reading units?", Applied Psycholinguistics 24 (2), 235-247.

GRAINGER J. et al. (2012), “Orthographic processing in Baboons (Papio papio)”, Science 336 (6078), 245-248. 
HARM M. W. \& SEIDENBERG M. S. (1999), "Phonology, reading acquisition, and dyslexia: Insights from connectionist models", Psychological Review 106 (3), 491-528.

Lallier M., ACHA J. \& CarReiras M. (2016), “Cross-linguistic interactions influence reading development in bilinguals: A comparison between early balanced French-Basque and SpanishBasque bilingual children", Developmental Science 19 (1), 76-89.

LANDERL K. et al. (2013), "Predictors of developmental dyslexia in European orthographies with varying complexity", Journal of Child Psychology and Psychiatry 54 (6), 686-694.

MANIS F. R. et al. (1996), "On the bases of two subtypes of developmental dyslexia", Cognition 58 (2), 157-195.

National Reading Panel (2000), Report of the National Reading Panel - Teaching Children to Read: An Evidence-Based Assessment of the Scientific Research Literature on Reading and its Implications for Reading Instruction, Washington (DC), National Institute of Child Health and Human Development.

PeEREMAN R. \& Content A. (1999), “LEXOP: A lexical database providing orthography-phonology statistics for French monosyllabic words", Behavior Research Methods, Instruments, and Computers 31 (2), 376-379.

Peereman R. \& Sprenger-Charolles L. (2018), "Manulex-MorphO, une base de données sur l'orthographe du français intégrant les morpho-phonogrammes ", Langue française 199. (ce volume)

PLAUT D. C. et al. (1996), “Understanding normal and impaired word reading: Computational principles in quasi-regular domains", Psychological Review 103 (1), 56-115.

RAU A. K. et al. (2015), "Effects of orthographic consistency on eye movement behavior: German and English children and adults process the same words differently", Journal of Experimental Child Psychology 130 (Suppl. C), 92-105.

RAYNER K. et al. (2001), "How psychological science informs the teaching of reading", Psychological Science in the Public Interest 2 (2), 31-74.

RUECKL J. G. et al. (2015), “Universal brain signature of proficient reading: Evidence from four contrasting languages", Proceedings of the National Academy of Sciences of the United States of America 112 (50), 15510-15515.

RuIz J.-P. et al. (2017), " GraphoGame : un outil numérique pour enfants en difficultés d'apprentissage de la lecture ", Approche Neuropsychologique des Apprentissages chez l'Enfant (A.N.A.E.) 148, 333-343.

SAKSIDA A. et al. (2016), "Phonological skills, visual attention span, and visual stress in developmental dyslexia”, Developmental Psychology 52 (10), 1503-1516.

SeIDENBERG M. S. \& McCleLLAND J. L. (1989), "A distributed, developmental model of word recognition and naming", Psychological Review 96 (4), 523-568.

SeYMouR P. H., ARo M. \& ERSKINE J. M. (2003), "Foundation literacy acquisition in European orthographies", British Journal of Psychology 94 (2), 143-174.

SHARE D. L. (1995), "Phonological recoding and self-teaching: Sine qua non of reading acquisition", Cognition 55 (2), 151-218.

SHARE D. L. (2008), "On the Anglocentricities of current reading research and practice: The perils of overreliance on an 'outlier' orthography", Psychological Bulletin 134 (4), 584-615.

SPRENGER-CHAROLLES L. (2017), " Une progression pédagogique construite à partir de statistiques sur l'orthographe du français (d'après Manulex-Morpho) : pour les lecteurs débutants et atypiques ", Approche Neuropsychologique des Apprentissages chez l'Enfant (A.N.A.E.) 148, 247-256. 
SPRENGER-CHAROLLES L. et al. (2003), "Development of phonological and orthographic processing in reading aloud, in silent reading, and in spelling: A four-year longitudinal study", Journal of Experimental Child Psychology 84 (3), 194-217.

SPRENGER-CHAROLLES L. et al. (2011), "Prevalence and reliability of phonological, surface, and mixed profiles in dyslexia: A review of studies conducted in languages varying in orthographic depth", Scientific Studies of Reading 15 (6), 498-521.

VAN ORDEN G. C. (1987), “A ROWS is a ROSE: Spelling, sound, and reading”, Memory \& Cognition 15 (3), 181-198.

WHITE S. et al. (2006), "The role of sensorimotor impairments in dyslexia: A multiple case study of dyslexic children", Developmental Science 9 (3), 237-255.

Wimmer H. \& GosWAMI U. (1994), "The influence of orthographic consistency on reading development: Word recognition in English and German children”, Cognition 51 (1), 91103.

ZIEgLeR J. C. \& Goswamı U. (2005), "Reading acquisition, developmental dyslexia and skilled reading across languages: A psycholinguistic grain size theory”, Psychological Bulletin 131 (1), 3-29.

ZIEGLER J. C. \& Goswamı U. (2006), "Becoming literate in different languages: Similar problems, different solutions", Developmental Science 9 (5), 429-436.

Ziegler J. C., Perry C. \& Coltheart M. (2000), “The DRC model of visual word recognition and reading aloud: An extension to German”, European Journal of Cognitive Psychology 12 (3), 413-430.

Ziegler J. C., VAN ORDEN G. C. \& JACOBS A. M. (1997), "Phonology can help or hurt the perception of print”, Journal of Experimental Psychology: Human Perception \& Performance 23 (3), 845-860.

ZIEGLER J. C. et al. (1996), "Statistical analysis of the bidirectional inconsistency of spelling and sound in French", Behavior Research Methods, Instruments, and Computers 28 (4), 504-515.

ZIEGLER J. C. et al. (1997), "What is the pronunciation for -ough and the spelling for $/ \mathrm{u} /$ ? A database for computing feedforward and feedback consistency in English", Behavior Research Methods, Instruments, and Computers 29 (4), 600-618.

ZIEGLER J. C. et al. (2001), "Identical words are read differently in different languages", Psychological Science 12 (5), 379-384.

ZIEGLER J. C. et al. (2003), “Developmental dyslexia in different languages: Language-specific or universal?”, Journal of Experimental Child Psychology 86 (3), 169-193.

ZIEGLER J. C. et al. (2008), "Developmental dyslexia and the dual route model of reading: Simulating individual differences and subtypes", Cognition 107 (1), 151-178.

ZIEGLER J. C. et al. (2010), “Orthographic depth and its impact on universal predictors of reading: A cross-language investigation", Psychological Science 21 (4), 551-559.

ZIEGLER J. C., PerRY C. \& ZorZI M. (2014), “Modelling reading development through phonological decoding and self-teaching: Implications for dyslexia", Philosophical Transactions of the Royal Society of London. B: Biological Sciences 369 (1634), 20120397. 


\title{
ABSTRACTS
}

\section{Johannes C. Ziegler, Cross-linguistic differences in learning-to-read}

This article summarizes the main research on cross-linguistic differences in the field of reading development. This research is at the origin of a universal theory of normal and impaired reading development and questions the narrow "Anglocentric" vision of reading. We present some of the findings that validated this theory in children, adults and bilinguals. Finally, we discuss the implications of this research for teaching and our understanding of impaired reading development.

Keywords : reading, learning-to-read, consistency, regularity, dyslexia

\section{RÉSUMÉS}

\begin{abstract}
Johannes C. Ziegler, Différences inter-linguistiques dans l'apprentissage de la lecture

Cet article résume les recherches sur les différences inter-langues dans le domaine de l'apprentissage qui sont à l'origine d'une théorie universelle de l'apprentissage de la lecture et qui remettent en question une vision trop " anglocentrique " de la lecture. Nous revenons sur les expériences qui ont validé cette théorie chez l'enfant, l'adulte et le bilingue. Enfin, nous discutons les implications de ces recherches pour l'enseignement de la lecture et la compréhension des troubles de la lecture.
\end{abstract}

Mots-clés : lecture, apprentissage, consistance, régularité, dyslexie 